
\section{BMJ Quality}

\title{
Improving Clinical Remission Rates in Pediatric Inflammatory Bowel Disease with Previsit Planning
}

\author{
Jeffrey R. Savarino, Jess L. Kaplan, Harland S. Winter, Christopher J. Moran, \\ Esther J. Israel
}

To cite: Savarino JR, Kaplan JL, Winter HS, et al. Improving Clinical Remission Rates in Pediatric Inflammatory Bowel Disease with Previsit Planning. BMJ Quality Improvement Reports 2016;5:u211063.w4361. doi:10.1136/bmjquality. u211063.w4361

Received 10 May 2016 Revised 15 July 2016 Published Online First 25 July 2016

CrossMark

MassGeneral Hospital for Children, United States of America

Correspondence to Jeffrey R. Savarino jsavarino@partners.org

\section{ABSTRACT}

Inflammatory Bowel Disease (IBD) is a chronic autoimmune inflammatory disease of the intestine which can lead to malnutrition, poor quality of life, and colon cancer. ${ }^{1-4}$ Although there is no cure for the disease, clinical remission is the primary goal. ${ }^{5}$ The Center for Inflammatory Bowel Disease at MassGeneral Hospital for Children (MGHfC) adopted a Previsit Planning (PVP) model to identify and discuss symptomatic patients prior to their appointments to identify specific issues that impact disease management. ${ }^{6-8}$ The Registry from ImproveCareNow (ICN), the international Quality Improvement Collaborative for the management of Crohn's Disease and Ulcerative Colitis in pediatric and adolescent patients, was used to capture information from each ambulatory visit and hospitalization. Using the Model for Improvement framework, the team began a weekly review and made care recommendations of patients with active disease who were cared for by one physician. Interventions were modified over multiple Plan-Do-Study-Act (PDSA) improvement cycles to increase the number of providers and to include patients with mild or moderate disease activity. ${ }^{9}$ Feedback from the providers regarding this process was elicited via a REDCap survey and the clinical remission rate was tracked using the ICN Registry. The clinical remission rate for the Center's patients increased from $77 \%(n=597)$ in September 2014 to 83\% $(n=585)$ in August 2015 and has been maintained. $78 \%$ of responding providers indicated that they found the PVP recommendations helpful "all of the time". One hundred percent who responded to the survey said that they have used at least one recommendation provided to them. PVP for management of a chronic disease in pediatrics is feasible, even in a high volume practice. This process at MGHfC has resulted in the improvement of clinical remission rate. PDSA cycles were used to document successes and failures to help guide the work. Ongoing expansion of this PVP practice to all providers continues with the anticipation of including input from patients and their families, as well.

\section{PROBLEM}

Inflammatory Bowel Disease (IBD), including Crohn's disease and ulcerative colitis, is a chronic gastrointestinal disorder. The management of pediatric patients with IBD has become increasingly more complex with an expanding repertoire of options for medical and surgical therapy. ${ }^{10-12}$ Currently, there is no cure for IBD and clinical remission rate is one of the major goals of therapy. Variations in management in a multi-provider and multisite practice model present many challenges to improvement work to increase the overall remission rates. In September, 2014 this Pediatric IBD center's remission rate was $77 \%$. The overall aim of this project was to increase the cohort remission rate to above $80 \%$ within one year. Previsit Planning (PVP) was instituted to accomplish this goal. A secondary goal of this work was to create a process assessed as useful by the providers.

\section{BACKGROUND}

The management of IBD is complex with multiple options for therapeutic intervention including corticosteroids, immunomodulators, biologics, and nutritional therapy. ${ }^{10-12}$ At times it is difficult to know when the appropriate time is to escalate or change therapy, and hesitation can lead to further complications. ${ }^{13}$ Reviewing patients' status, updating their health maintenance issues, and planning the next phase of their care during the time allotted for a follow up visit is challenging. The Division of Gastroenterology and Nutrition at MassGeneral Hospital for Children (MGHfC) is a large division within an academic medical center, comprised of twenty-three attending physicians, three nurse practitioners, and six fellows with different backgrounds and styles of practice, caring for over 700 children and young adults with IBD. Collaboration is hindered because providers practice in multiple sites and are rarely together to discuss patient care. Guideline algorithms for care rarely address the 


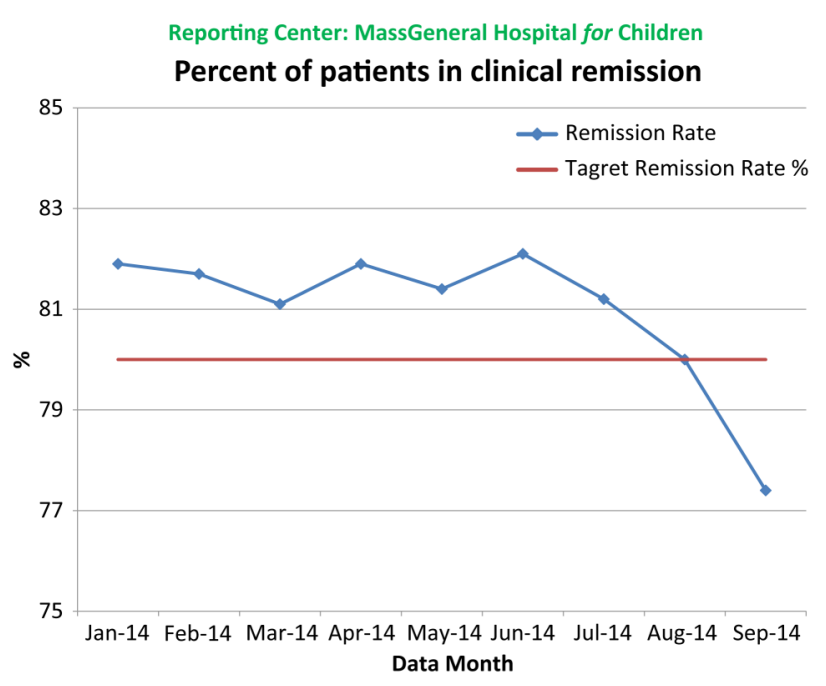

nuances of care delivery to the individual patient.

The Pediatric IBD Center at MGHfC is a member of ImproveCareNow (ICN), an international quality improvement collaborative and learning health community focused on improving care and outcomes for children and adolescents with $\mathrm{IBD}^{6}$ and enhancing collaboration among centers and between providers and patients. Centers that are part of the network enter data for each registered patient visit, including physician global assessment (PGA), medications, lab results, stooling pattern, as well as nutrition and growth status. The primary goal of ICN is to improve remission rate as assessed by the PGA. ${ }^{14}$ The network provides resources to track clinical outcomes and guide clinical improvement efforts. ICN centers are encouraged to share data and workflows to improve care across all centers. One of the pillars of ICN is the development and implementation of comprehensive, routine PVP for patients with upcoming visits. ${ }^{6}$ Automated PVP reports are generated by the ICN Registry which includes data based on the providers' documentation at the previous outpatient visits with some recommendations based on Model IBD Care Guidelines. ${ }^{15}$ For each visit, the physician records a PGA, nutrition and growth status, corticosteroid use, and hospitalization history. The Registry calculates a Care Stratification Score (CSS) to identify at-risk individuals. ${ }^{16}{ }^{17}$ On a scale of $0-12$, where zero indicates a patient is doing well in all parameters, and 12 represents a patient who is experiencing a severe disease flare, the CSS score is used as a measure of illness severity.

PVP has been used to improve quality of care provided to patients with chronic diseases. Implementation of a PVP model in the care of adults with Type II Diabetes Mellitus helped to identify patients who were due for lab orders or health maintenance exams, and the process allowed for patient-specific decision making. In this model, clinic staff provided physicians with individualized information for each patient at the time of visit; physicians were reminded of outstanding tests and

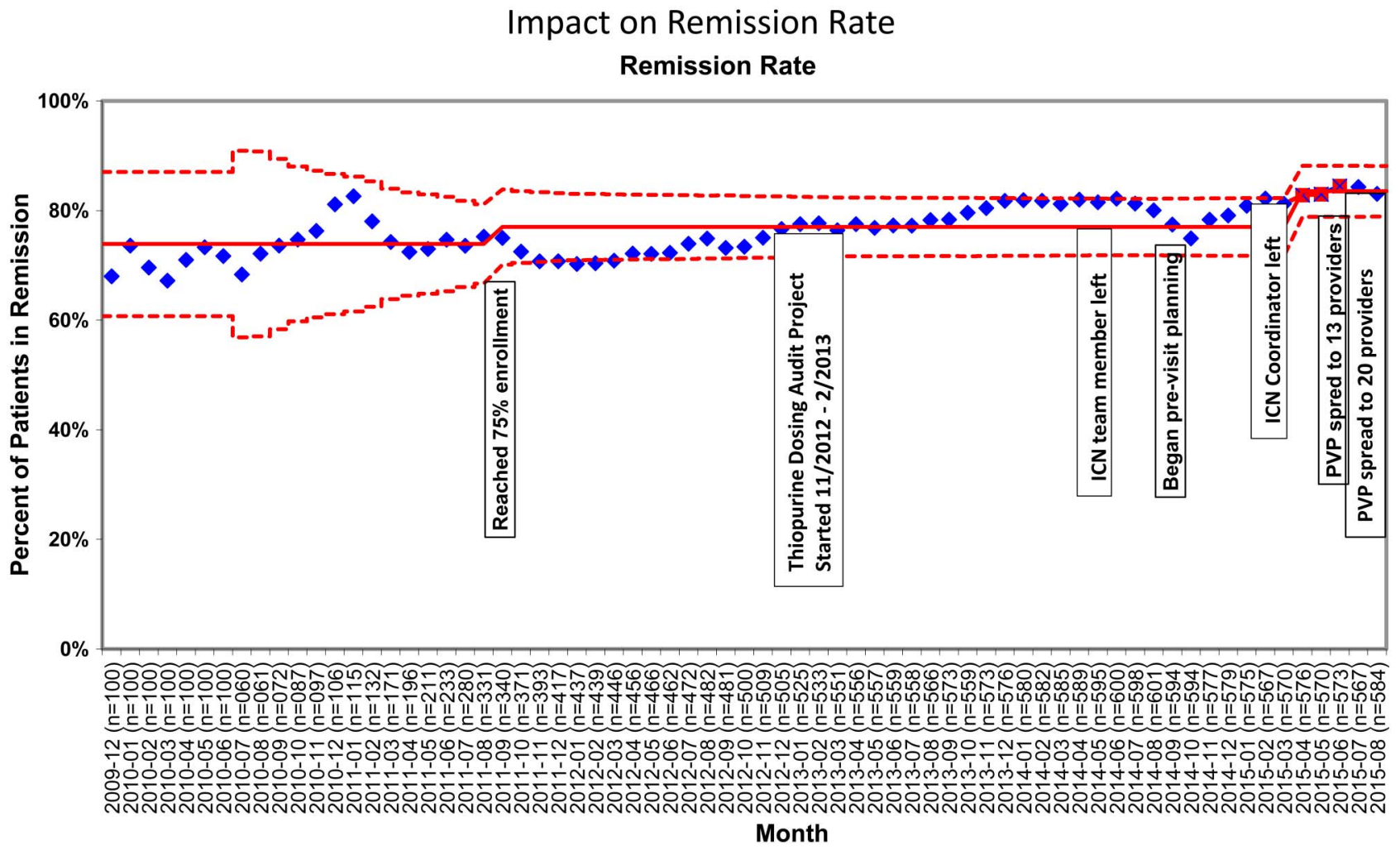

- Remission Rate

Mean Remission Rate

- - Control Limits 
appointments and were alerted if outcome goals were not met. ${ }^{7}$ The AMA estimates that PVP can save both physician and clinic staff up to 30 minutes per day, a potential savings of $\$ 26,400$ annually. $^{8}$

\section{BASELINE MEASUREMENT}

The ICN Registry was used to capture information from each visit for all enrolled IBD patients. In the Spring of 2014, the remission rate, which had increased to above $80 \%$, started to decrease to a low of $77 \%(\mathrm{n}=597)$ by September, 2014. No specific cause of this decline was identified. Efforts were therefore re-directed at that point to improve the remission rate with the institution of PVP.

\section{DESIGN}

The goal was to use the Model for Improvement to design a PVP process that worked in this particular work environment. Active disease was used as a criterion for PVP to keep the number of patients per discussion manageable. The core team consisted of attending physicians, nursing, and a clinical research coordinator (CRC). In anticipation of each weekly meeting, the CRC identified all IBD patients to be seen the following week by the staff physicians. The core team discussed each patient designated for review. The review included, but was not limited to, IBD diagnosis and disease phenotype, growth status, recent diagnostic testing, health maintenance information (vaccine status, eye exam, vitamin D levels, etc.), medication history and current therapy, and recent hospitalizations or visits to an emergency department. The core team made recommendations on how to alter the patient's treatment regimen with a goal towards improving their disease activity. These recommendations were recorded and sent to the patient's primary gastroenterologist at the conclusion of the meeting. PDSA cycles were used to monitor changes implemented to improve and optimize the PVP process to make the largest impact on remission rate.

\section{STRATEGY}

The goal of this activity was to improve care and increase the remission rate of the division's IBD population. PVP started with one physician with the plan to include all physicians who take care of patients with IBD and were willing to receive input from the team. PVP started with the sickest of patients with the intent of expanding to all patients who were not in sustained remission. Rapid improvement cycles were aimed at ramping up to these goals.

PDSA Cycle 1 and 2: The CRC reviewed one provider's schedule for the following week and identified all ICN-enrolled patients using the Study Log. An automated PVP report was generated for each ICN patient ( $\mathrm{n}=22$ patients, 5.5/week) from the ICN Registry. Each patient with a Care Stratification Score $>8$ was discussed by the core team, looking for areas where change could bring about remission. Suggestions were then forwarded to the primary gastroenterologist by the CRC at the conclusion of the PVP meeting. It was hoped that physician would review the suggestions and consider implementing them at the patients' visits the following week. No patients met the criteria of a CSS $>8$ so the PVP process was expanded to another provider in Cycle $2 \quad(n=12$ patients, 6/week). That being said, the assessment of these cycles was that these were extremely complicated patients and the recommendations from the PVP meeting had been discussed previously and did not contribute significantly to the overall care of the patients. The PVP process was therefore expanded to include patients with lower CSS scores.

PDSA Cycle 3: In an attempt to expand the number of patients reviewed, the same two providers were included in the following cycle, but all IBD patients of these providers, regardless of disease activity status, were assessed. The core team was expanded to include four attending physicians in this cycle to get a wider perspective and to allow for the regular absences of providers secondary to other activities. There was a sense that these meetings were productive and there appeared to be time available to allow for more patients to be discussed, so the process was expanded to more providers' patients.

PDSA Cycles 4-7: Five providers' schedules were reviewed during cycle 5 and expanded by one additional provider for each of the next two cycles, PDSA cycle 6 and 7, with the ultimate goal of providing PVP support to all patients with IBD. However, during these cycles it became apparent that there were too many patients to be discussed and it was not worth extensively discussing the management of those patients with a CSS of 0 as they were already in remission so discussion would not help reach the goal of increasing remission rate. The evaluation of these cycles was that there was just not enough time to pull all of these data on the patients who were in remission while also sharing the team's collective wisdom related to those patients with higher care stratification scores. The ultimate goal was to expand to all of the patients in the practice to improve remission rate, so it was decided that those with scores $<$ or $=2$ would no longer be discussed.

PDSA Cycle 8-9: The CRC reviewed seven providers' schedules for the following week in the following cycle, just focusing on patients with CSS scores $>2$. This brought the average number of patients discussed at a given PVP meeting to 4 to 5 , and it was determined that there was time to discuss more patients. Therefore, patients from an additional provider were discussed in PDSA Cycle 9, bringing the total number of providers whose patients underwent the PVP process, to 8.

PDSA Cycle 10-13: PDSA cycle 10 included the patients of the same eight providers, but with a CSS $>2$, or equal to 2 with persistent disease activity during the last two clinic visits. The goal of this PDSA cycle was to determine whether the volume of patients to be included with these criteria would still be manageable in the PVP hour as they were contributing to the lower 
remission rate with their persistent activity scores. Patients from one additional provider were discussed in PDSA Cycle 11 with the same criteria for inclusion and then expanded to 13 providers so that PVP would be available to more patients with active disease. Comments were elicited from the providers at this point via an anonymous REDCap survey to assess the provider experience with the PVP process. The survey showed that a number of providers expressed interest in getting the recommendations more proximate to the day of the visit so the next cycle responded to this preference in hopes of greater acceptability of the recommendations.

PDSA Cycle 13-15: The patients of the same 13 providers were included in this next cycle. Instead of communicating immediately after the PVP meeting held one week prior to any of these visits, a Microsoft Outlook function was used to schedule communication to be sent to each patient's primary gastroenterologist on the morning of the anticipated visit. The review was expanded to 14 providers in the next cycle and eventually to 17 providers. At this point, an average of 7 patients were being discussed weekly, with a range of 2 to 13 .

\section{RESULTS}

Two different measures were tracked to monitor success of the PVP initiative. The first measure was the primary endpoint of the clinical remission rate. Changes in the clinical remission rate were tracked from before the implementation of PVP in September 2014 until the end of August 2015. The cohort remission rate increased from $77 \%(n=597)$ to $83 \%(n=585)$. Plotting the data on a control chart identified that there was a special cause contributing to the improvement of this quality measure; no other specific quality improvement initiatives directed at improving disease activity scores nor major changes in IBD treatment patterns were implemented and sustained during this time period.

Receiving feedback from the providers about the relevance of the recommendations was helpful and constituted evaluation of the secondary goal. An anonymous survey was sent out during PDSA Cycle 12 to all fourteen attending physicians whose patients were identified for review. Nine physicians responded to the survey. Of the respondents, $78 \%$ indicated that they found the PVP recommendations helpful "all the time", while $22 \%$ respondents indicated that they found the recommendations helpful "most of the time". $100 \%$ of respondents said that they have used at least one PVP recommendation made by the ICN team.

\section{LESSONS AND LIMITATIONS}

There were a number of challenges that were identified during the implementation of PVP. First, time was the factor limiting the development and expansion of the PVP process. The improvement team needed to have the time to review patients' charts and to make recommendations. Providers receiving the recommendations had to take the time to read and consider them in order for PVP to be meaningful. The team learned that with a dedicated hour to PVP once a week they were able to expand the process to a considerable number of at-risk patients and make a meaningful change. Buy-in was attained from providers by starting the initiative slowly with one provider's patients and slowly ramping up; other physicians were able to see the benefit of PVP and were more open to being involved in the process. The sustainability of this project relied on the allocation of time dedicated to PVP by the core team and continued support from division physicians. This was achieved by blocking time from the schedule of each core team physician, ensuring that providers always had a regular time to meet for PVP. Dissemination of recommendations occurred via secure e-mail on the morning of the patient's clinic visit, which required division gastroenterologists to have the time to check their e-mail prior to the start of their clinic day. Thus far, most physicians have worked this process into their regular clinic workflow, but any deviation could compromise the sustainability of the PVP project. Additionally, without research coordinator or support, it is likely that this process would not be sustainable, as PVP meeting planning and recommendation dissemination is largely reliant on the CRC or some other designated individual to coordinate the activity. While PVP has been adopted successfully at MGHfC, a dramatic improvement in remission rate may not be repeatable at other institutions with different patient cohorts, clinic infrastructure, and barriers to care. However, many other ICN centers have shown that it is possible to adopt a regular PVP process. Further analysis of how PVP has impacted remission rate at other ICN centers would determine how PVP could be applied to other clinical settings.

The PVP process was initially hampered by out of date information. There was a lag between a patient's clinic visit and entry of the visit information into the ICN Registry. It was found that this lag led to the core team discussing patients whose IBD was no longer active as interventions had already been made, while missing patients who had recently developed symptoms. This made the process ineffective, but through PDSA cycles, the core team was able to recognize this problem and improve data entry workflow to enhance the quality of recommendations that the core team could make.

An analysis of cost savings due to implementation of PVP has not yet been calculated. However, based on analyses in other disease models, it can be predicted that PVP in IBD can contribute to cost savings by optimizing staff workflows, decreasing comorbidity and complications, and improving achievement of recommended clinical values. ${ }^{78}$ Attainment of these improvements in a pediatric IBD PVP model may reduce IBD-related admissions, prevent unnecessary testing and procedures, and improve therapy to further reduce costs. A formal analysis of cost savings is necessary to completely evaluate the effect of the implementation of PVP at MGHfC. 


\section{CONCLUSION}

PVP for management of a chronic disease in pediatrics is feasible, even in a high volume practice. Initiation of this process at MGHfC has resulted in the improvement of care provided to children and adolescents with IBD. The primary goal of increasing clinical remission rate from $77 \%$ to greater than $80 \%$ within one year of initiating PVP was achieved. PDSA cycles were used to slowly ramp up efforts and documented successes and failures to decide where to focus future endeavors. Providers receiving these clinical recommendations indicated in an anonymous survey that they had reviewed and used these suggestions to guide their clinical practice. Most providers felt that these recommendations were helpful all of the time, which is important because many providers receive recommendations regarding several of their patients each week; the high frequency of PVP recommendations did not detract from the quality of the process. This suggested that the secondary goal of creating a useful and efficient PVP process was achieved. Initiation of PVP has coincided with a sustained improvement in remission rate for pediatric IBD at MGHfC. Further review and expansion of this practice to all providers continues and the core team hopes to include input from surgical, nutrition, mental health, and social work colleagues, as well as including parent and patient involvement in the process.

Acknowledgements The authors would like to acknowledge the quality improvement consultants from ImproveCareNow, whose expertise was integral to the design, implementation, and evaluation of our Previsit Planning process. This work was made possible through the generous support of Carroll and Bob Pierce.

\section{Declaration of interests None detected}

Ethical approval The intent of this work was to improve the quality of care provided to our IBD patients and it was not a study on our patients, per se.

Open Access This is an open-access article distributed under the terms of the Creative Commons Attribution Non-commercial License, which permits use, distribution, and reproduction in any medium, provided the original work is properly cited, the use is non commercial and is otherwise in compliance with the license. See:

- http://creativecommons.org/licenses/by-nc/2.0/

- http://creativecommons.org/licenses/by-nc/2.0/legalcode

\section{REFERENCES}

1. Mijac DD, Jankovic GLJ, Jorga J, Krstic MN. Nutritional status in patients with active inflammatory bowel disease: Prevalence of malnutrition and methods for routine nutritional assessment. Eur J Intern Med. 2010 Aug;21:315-9.

2. Loonen HJ, Grootenhuis MA, Last BF, Koopman HM, Derkx HH Quality of life in paediatric inflammatory bowel disease measured by a generic and a disease-specific questionnaire. Acta Paediatr. 2002;91:348-54.

3. Devroede GJ, Taylor WF, Sauer WG, et al. Cancer risk and life expectancy of children with ulcerative colitis. N Engl J Med. 1971 Jul;285:17-21.

4. Triantafillidis JK, Kosmidis P, Nasioulas G. Colorectal cancer and inflammatory bowel disease: epidemiology, risk factors, mechanisms of carcinogenesis and prevention strategies. Anticancer Res. 2009 Jul;29:2727-37.

5. Homan M, Baldassano RN, Mamula P. Managing complicated Crohn's disease in children and adolescents. Nat Clin Pract Gastroenterol Hepatol. 2005 Dec;2:572-9.

6. Crandall W, Kappelman M, Colletti M, et al. ImproveCareNow: the development of a pediatric inflammatory bowel disease improvement network. Inflamm Bowel Dis. 2011 Jan;17:450-7.

7. Peterson KA, Radosevich DM, O'Connor PJ, et al. Improving diabetic care in practice. Diabetes Care. 2008 Dec;31:2238-2243.

8. Sinsky C. Practice transformation series: pre-visit planning [Internet]. [Place unknown]: American Medical Association. 2014 [Cited 2016 Mar 21]. https://www.stepsforward.org/modules/pre-visit-planning.

9. Cleghorn GD, Headrick LA. The PDSA cycle at the core of learning in health professions education. J Comm J Qual Improv. 1996 Mar;22:206-12.

10. Girardin M, Manz M, Manser C, et al. First-line therapies in inflammatory bowel disease. Digestion. 2012;86(suppl 1):6-10.

11. Prefontaine E, Macdonald JK, Sutherland LR. Azathioprine or 6-mercaptopurine for induction of remission in Crohn's disease. Cochrane DB Syst Rev. 2009 Oct;7:CD000545.

12. Lee YM, Kang B, Lee Y, Kim MJ, Choe YH. Infliximab "top-down" strategy is superior to "step-up" in maintaining long-term remission in the treatment of pediatric Crohn's disease. J Pediatr Gastroenterol Nutr. 2015 Jun;60:737-43.

13. Burger $D$ and Travis $S$. Conventional medical management of inflammatory bowel disease. Gastroenterology. 2011 May;140:1827-37.

14. Physician global assessment [Internet]. [Place unknown]: ImproveCareNow. [Cited 2016 Mar 21]. https://improvecarenow.org/ care-providers/physician-global-assessment.

15. Model IBD Care-a Guideline for Consistent Reliable Care [Internet]. [Place unknown]: ImproveCareNow [Cited 2016 July 8]. https:// d3n8a8pro7vhmx.cloudfront.net/improvecarenow/pages/283/ attachments/original/1464375801/Model_IBD_Care_Guideline_2016. pdf?1464375801

16. Glaseroff A, Lindsay A. Building an intensive primary care practice [Internet]. [Place Unknown]: American Medical Association. [Cited 2016 Mar 21]. https://www.stepsforward.org/modules/ intensive-primary-care.

17. Risk-Stratified Care Management [Internet]. [Place Unknown]: American Academy of Family Physicians. [Cited 2016 Mar 21]. http://www.aafp.org/practice-management/transformation/pcmh/ advanced/rscm.html?cmpid=_van_596. 